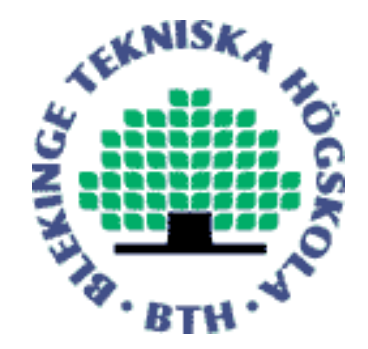

Copyright @ 2008 IEEE. Citation for the published paper:

Isaksson, Lennart; Fiedler, Markus

"Seamless Connectivity in WLAN and Cellular Networks with Multi Criteria Decision Making”

3rd EuroNGI Conference on Next Generation Internet Networks (NGI 2007)

Trondheim, Norway, 21-23 May 2007, Pages: 56 - 63

This material is posted here with permission of the IEEE. Such permission of the IEEE does not in any way imply IEEE endorsement of any of BTH's products or services Internal or personal use of this material is permitted. However, permission to reprint/republish this material for advertising or promotional purposes or for creating new collective works for resale or redistribution must be obtained from the IEEE by sending a blank email message to pubspermissions@ieee.org.

By choosing to view this document, you agree to all provisions of the copyright laws protecting it. 


\section{Seamless Connectivity in WLAN and Cellular Networks with Multi Criteria Decision Making}

\author{
Lennart Isaksson \\ Department of Telecommunication Systems \\ School of Engineering \\ Blekinge Institute of Technology \\ Karlskrona, Sweden \\ Email: lennart.isaksson@bth.se
}

\author{
Markus Fiedler \\ Department of Telecommunication Systems \\ School of Engineering \\ Blekinge Institute of Technology \\ Karlskrona, Sweden \\ Email: markus.fiedler@bth.se
}

\begin{abstract}
The evolution of today's wireless and mobile technologies and personalized hand-held devices has increased the challenge to handle the trade-off between mobility, performance and cost. The user simply needs to be Always Best Connected (ABC). To make this happen, different types of criteria reflecting relevant characteristics of wireless and mobile access networks such as WLAN, UMTS and GPRS have to be established and judged. In this paper the criteria are divided into three main groups, performance, cost and accessibility. The criteria are fed into a Multi-Criteria Decision Making (MCDM) tool which is able to deliver a ranking of network links in order to become ABC. Finally, a case study with two different Generic Services (GSs) illustrates the decision making process and its outcomes in different situations.
\end{abstract}

\section{INTRODUCTION}

Personalized communication and information technology has steadily grown in importance during the recent years. Today, a user has the ability to choose the way to communicate via fixed, wireless or mobile access network. Instead of using one telephone connected to only one provider, one has the option to choose between several services and providers. However, an average user does not necessarily comprehend the offerings and implications of different access networks for different Generic Services (GSs) such as streaming, messaging and interactive [1]. So, the challenge is to choose an acceptable network link for the selected service so that a reasonable performance-cost-accessibility compromise is yielded. In highly dynamic wireless and mobile environments, involving varying numbers of users and volatile transmission conditions, the characteristics of streams of data packets change all the time, which imposes the need of end-to-end monitoring. Also, the amount of simultaneous users which could be connected to the same Access Point (AP) or base station do have some major impacted on the overall performance. Moreover, the problem with interference in the $2.4 \mathrm{GHz}$ Industrial Scientific and Medical (ISM)-band, shared amongst Wireless Local Area Network (WLAN), ZigBee, Bluetooth and microwave ovens, needs to be addressed [2].

No matter of the conditions and obstacles, the main goal for any end-user is to be Always Best Connected (ABC), i.e. "he or she is not only always connected, but also connected through the best available device and access technology at all times" [3]. "Best" refers to a set of criteria. In the following, a particular criterion known beforehand and not to be changed during a session is defined as a static criterion. If the changes happen within a session, e.g. due to traffic conditions, we speak of a dynamic criterion. Based on given and monitored information, a decision is made from the end-user perspective. This decision and the related parameters depend amongst others on the type of GS to be used.

Given the growing mobility of users and the volatility of conditions, necessary changes of access networks need to be carried out during ongoing sessions. This is not enabled by the Internet Protocol (IP) being the most-spread networking protocol today. A change of network means a change of the IP address and thus breaking the session, entailing data loss and the necessity to start over. The dream scenario from the end-user point of view is, however, seamless communications implying switching or roaming between access networks (also called vertical handover) which should be hardly noticed by the end-user.

Most of the approaches to seamless communications are based on Mobile IP (MIP), an extension of IP that requires additional agents in the network. Bakshi et al. [4] are looking at cost effective solutions during active handover for WLAN with Transmission Control Protocol (TCP). Also, Indulska et al. [5] investigate the context-aware vertical handover between WLAN and $3 \mathrm{G}$ networks. The prototype was developed to demonstrate vertical handover between WLAN and General Packet Radio Service (GPRS) and vice versa using streaming Joint Photographic Experts Group (JPEG) video. Their results show low delays and low packet losses due to a disconnection and a reconnection, which is mandatory in a Mobile IP scenario. Floroiu et al. [6] have looked at seamless handover using both User Datagram Protocol (UDP) and TCP in Mobile IP for WLAN / GPRS. The results show that the Round Trip Time (RTT) is one of the important parameters indicating 
performance degradation.

True seamless communications does not allow for delays of losses during the handover process. Åhlund et al. [7] extend MIP to Multihomed Mobile IP (M-MIP) using simultaneously connected links, but still requiring extra functionality in the network. In [8], we presented a true end-to-end solution for seamless communications, based on a so-called Network Selection Box (NSB) and leaving both IP and the network untouched.

This paper focuses on the decision process that governs the change of networks. We define a framework for this decision process and concentrate on the Multi-Criteria Decision Making (MCDM) tool, based on the Analytic Hierarchy Process (AHP). Hou et al. [9] use a decision making algorithm based entirely on fuzzy logic which belongs to the group of dynamic programming. Qingyang et al. [10] describe a network selection scheme for an integrated cellular/WLAN system. In this system two decision method are used, the AHP and the Grey Relational Analysis (GRA), both targeting ABC and thus the end-user. Åhlund et al. [7] describe a model of handling interference in an access point using the Signal-toNoise Ratio (SNR). The parameters of the criteria used are not related to any GS in contrary to our approach.

The remainder of the paper is organized as follows: In Section II, our decision framework for seamless communications is defined. Section III presents the criteria under consideration. In Section IV the decision model Multi-Criteria Decision Making (MCDM) and the Analytic Hierarchy Process (AHP) are described. This is followed by an outline of metric value translations to be carried out ahead of the decision process in Section V. In Section VI a case study of two types of GSs, namely streaming and messaging, in different situations is given. Finally, in Section VII, the conclusions are presented.

\section{Decision Framework for Seamless COMMUNICATIONS}

Fig. 1 sketches the decision framework of our seamless communication concept. Data and control streams are indicated by thick and thin lines, respectively. Seamless communications needs to address three main components as follows: (1) A Generic Service such as Streaming Service (SS), Messaging Service (MS) and Interactive Service (IS) running in a certain environment (smartphone, PDA, laptop, etc.) puts specific demands on the network selection for $\mathrm{ABC}$, which is taken care of by (2) Seamless Connectivity, the core functionality. It exchanges management information with the application, extracts important information from the data flow and sends the latter to (9) Switch Control Unit, switching between the well-known hidden technologies W(LAN), U(MTS) and G(PRS). The commands for these switches are generated along the control path (3)-(8): (3) Criteria reflect the needs of the GS. They thus can look quite differently and/or be of different importance for different GSs. A distinction is made between inner (performance) and outer (cost, accessibility) criteria. The chosen criteria will be further explained in the next section. (4) Metric values for these criteria are taken from a-priori [11]

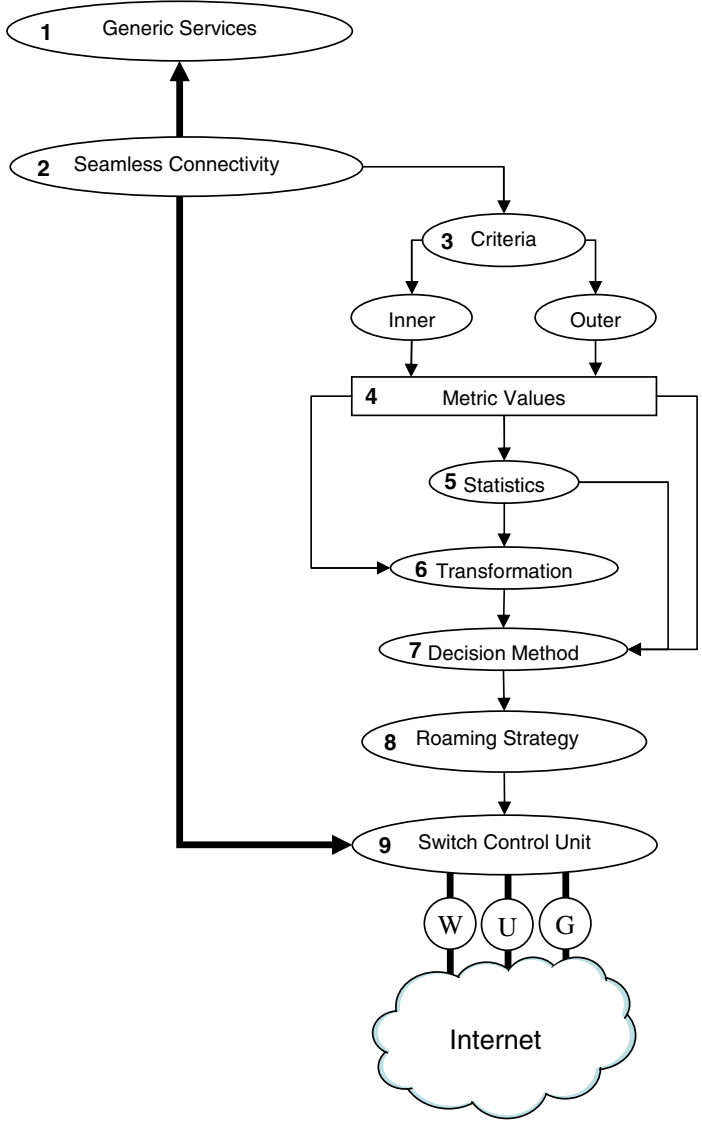

Fig. 1. Decision framework for seamless communications

or from on-line measurements. In both cases, they have to be linguistically interpreted according to input requirements from the decision method. This and the different paths between (4) and (7) shown in Fig. 1 will be detailed in Section V. (5) There are several ways of processing data into statistics, such as averages, standard deviations, histograms, or autocorrelation coefficients [11], depending on what is adequate for the final decision. (6) Metric values need to be mapped into a multipoint scale suitable for decision making, which is called transformation. This could be done in different ways, e.g. by comparisons against thresholds or by using Fuzzy Set Theory (FST), which is called an "approximate reasoning" process. (7) The decision method collects multi-point scale data from the previous stages in order to prepare the choice of the network, which preferably has to be carried out in real time while the service is going on. We will take a deeper look at this step in Section IV. (8) The roaming strategy finally generates the control signals for the switch control unit, taking also direct user interference into account.

\section{CRITERIA}

The subjective quality combines the human perception and the objective quality [12]. The objective quality consists of four components, transport quality being one of them. The transport could be done via any wireless system, e.g. Universal Mobile Telecommunications System (UMTS), GPRS, 
or WLAN. Each wireless system has its own characteristics that need to be considered when working toward ABC. Different criteria should be considered, e.g. initial delays of a connection, link capacities, data loss, throughput properties, the payment model for the subscription, coverage etc. Not every criterion has the same importance for every service, as different parameters affect the perception of different GSs in different ways.

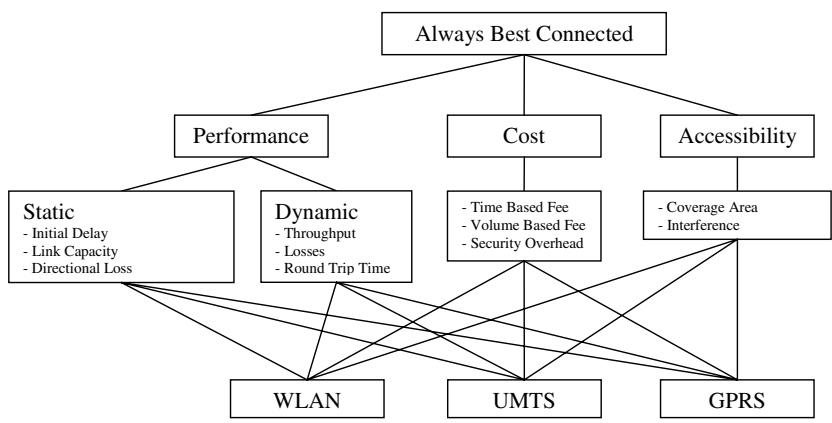

Fig. 2. Hierarchy of criteria for seamless communications, targeting Always Best Connected

Fig. 2 presents a hierarchy of criteria with the goal of ABC. To be able to reach this goal three groups have been defined: performance, cost and accessibility: (1) Performance refers to inner criteria related to properties of data transportation as observed from a-priory measurements (static) or from online end-to-end monitoring (dynamic) [8]. Static performance parameters used in our approach are:

- Initial Delays (ID). The setup time for a connection takes time, in some systems more than in others. According to Fiedler et al. [11], initial delays for GPRS are mostly found within some few seconds, while for UMTS, initial delays up to seven seconds were not uncommon. WLAN connectivity, on the other hand, is perceived as responding instantaneously.

- Link Capacity (LC). This parameter can span several orders of magnitude: GPRS can reach $57 \mathrm{kbps}$, UMTS $384 \mathrm{kbps}$ and WLAN 54 Mbps.

- Directional Loss (DL). Packet loss for UMTS and GPRS can look very different in different directions. While it can be very low in the uplink direction when a feedbackproviding send function is used in the terminal [11], it can be arbitrarily high especially for GPRS if there is a capacity mismatch in the downlink direction. Unfortunately, the allocation strategy of GPRS is hidden to the user.

As dynamic performance parameters, we use:

- Throughput. Ideally, the temporal characteristics of data streams should not be changed by the network inbetween. Throughput statistics employing short averaging intervals are easily measured and allow for the detection of anomalies stemming from transmission and congestion problems [11]. Links inducing heavy throughput variations should be avoided especially in case of streaming services.

- Losses. For any kind of service, high observed message or packet losses provide indications to search for another, more stable network link.

- Round Trip Time (RTT) provides indications about the cumulative delay in both directions that has a strong influence on the perception of interactive services. Oneway delays may be even more interesting, but hard to measure due to time synchronization problems.

Regarding cost, we focus on:

- Time-Based Fee (TBF) and Volume-Based Fee (VBF), which depend on the type of subscription. There is a trend toward time-based flat rate subscriptions. A VBF can become very expensive for streaming services.

- Security. Its impact is considered through its throughput overhead, as in general, secured data packets grow in length. This negative effect of adding security features grows as capacity decreases, which speaks against GPRS and in favor of WLAN.

Finally, accessibility is addressed by the following parameters:

- Coverage Area (CA), which depends on frequency bands and power levels used. UMTS and the GPRS cover larger areas compared to the WLAN designed for local scenarios.

- Interference is mainly an issue for WLAN (shares the $2.4 \mathrm{GHz}$ ISM-band with other technologies) and to a certain extent even for UMTS (through the Code-Division Multiple Access).

\section{Decision Method}

A well known set of methods for decision making is Multi-Criteria Decision Making (MCDM) or Multi Attributive Decision Making (MADM) based on the Analytic Hierarchy Process (AHP). It is a general class of models which deal with decision problems in the presence of a number of decision criteria. The final decision is highly dependent on the preferences of the decision maker and must be a compromise. However, the preferences we expressed as quantities based on measurements and judgments, which will be illustrated in Section V.

\section{A. Analytic Hierarchy Process}

The AHP method will be used to select the best link alternative with Quality of Service (QoS) level in mind and was originally proposed by Saaty in [13] to support decision in Management Science [14]; further references by Saaty are [15]-[19]. It has also been used in several software engineering initiatives [20]-[23], and several programs have been developed using AHP as a foundation [24]-[28].

The AHP method for solving decision problems includes five major steps:

- Step 1: Construct the hierarchical structure by breaking down and decomposing the decision problem into several decision elements.

- Step 2: Create the input values by pair-wise comparisons of decision elements. 
- Step 3: Estimate the relative weights of the decision elements.

- Step 4: Check for consistency.

- Step 5: Synthesize the priorities and combine the relative weights to determine the final set of ratings for and thus a ranking of the different decision alternatives.

\section{B. Step 1}

The critical part of decision making is the structuring. This includes the decomposition of the problem into several elements according to their characteristics. In order to model complex decisions correctly and efficiently the following guidelines should be kept in mind:

1) Try to cluster elements so that they include elements that are comparable, or that do not differ too much from each other. This was taken into account when defining the hierarchy shown in Fig. 2. Its basic form consists of a hierarchy structure with the goal at the top level, inbetween the clustered criteria, and last the alternatives.

2) Do not include more than nine elements in any set. Experiments have shown that it is challenging for human beings to deal with more than nine factors at one time and this can result in less accurate priorities, demonstrated in [29]. Moreover, as the number of elements being compared increases the measure of inconsistency decreases so slowly that there is insufficient space for improving the assessment as well as the consistency.

3) When it comes to subjective quality, Miller [30], [31] as early as in 1956 stated an upper limit of short-term memory based on human capacity. It is recommended to use a seven-(plus-minus-two-)point scale [30].

\section{Step 2}

The judgments in the AHP are made in pairs $a_{i j}$, relating the importance of criterion $i$ to that of criterion $j$. The scale used is represented by the intensities between each other according to the fundamental scale. The fundamental scale is validated according to effectiveness and theoretical justifications according to Saaty [13]. The scale consists of nine levels. To make it even easier to judge, one can use a more restricted scale with five levels: 1 is equal importance, 3 is moderate importance, 5 is strong importance, 7 is very strong or demonstrated importance and 9 is extreme importance.

First, the criteria are compared pair-wise with respect to the goal. A $i \times j$ matrix, denoted as $\mathbf{A}$, is created using the comparisons with elements $a_{i j}>0$, indicating the importance of criterion $i$ relative to criterion $j$ as shown in Equation (1). Obviously, $a_{i j}=1$ when $i=j$, while $a_{j i}=1 / a_{i j}$, which reflects the reciprocal importance of criterion $j$ relative to criterion $i$.

$$
\mathbf{A}=\left[\begin{array}{ccccc}
a_{11} & a_{12} & a_{13} & \cdots & a_{1 j} \\
a_{21} & a_{22} & a_{23} & \cdots & a_{2 j} \\
\vdots & & & & \\
a_{i 1} & a_{i 2} & a_{i 3} & \cdots & a_{i j}
\end{array}\right]
$$

\section{Step 3}

After constructing the matrix of comparison, the next step is to determine the weights of the criteria, in which $w_{i}$ is the weight of objective $i$ in the weight vector $\mathbf{w}=$ $\left[w_{1}, w_{2}, \cdots, w_{n}\right]$ for $n$ criteria. The objective is to recover vector $\mathbf{w}$ from matrix $\mathbf{A}$ by finding the solution for some value $u$ of

$$
\mathbf{A} \cdot \mathbf{w}^{T}=u \cdot \mathbf{w}^{T} .
$$

The matrices $\mathbf{A}^{\mathrm{Ck}}$ contain judgments of the importance of criteria against each other per group $k \in\{1=$ performance, 2 = cost, $3=$ accessibility $\}$. The matrices $\mathbf{A}^{\mathrm{A} k l}$ contain judgments of the importance of attributes per criterion $k$. Index $k$ and index $l$ together form the following combinations: $11=$ Initial Delays (ID), 12 = Link Capacity (LC), 13 = Directional Loss (DL), $14=$ throughput, $15=$ losses, $16=$ Round Trip Time (RTT), 21 = Time-Based Fee (TBF), 22 = Volume-Based Fee $(\mathrm{VBF}), 23$ = security, $31=$ Coverage Area $(\mathrm{CA}), 32=$ interference.

In order to determine $w_{i}$ a numerical solution is used [13] that starts with normalizing each column $j$ in $\mathbf{A}$ such that

$$
a_{i j}^{\prime}=\frac{a_{i j}}{\sum_{i=1}^{n} a_{i j}} .
$$

Next, each row $i$ in $a^{\prime}$ is summarized into a vector with elements

$$
a_{i}^{\prime \prime}=\sum_{j=1}^{n} a_{i j}^{\prime} .
$$

Finally, the vector $\mathbf{w}$ is obtained as

$$
w_{i}=\frac{a_{i}^{\prime \prime}}{\sum_{j=1}^{n} a_{j}},
$$

where $w_{i}$ is the weight of criteria $i$ in the weight vector.

\section{E. Step 4}

The next step is to check for consistency. According to [13] four steps are used as follows:

1) Compute $\lambda_{\max }$, which is the largest eigenvalue of matrix A.

2) Compute the Consistency Index $\mathrm{CI}(n)=\left(\lambda_{\max }-\right.$ $n) /(n-1)$.

3) Compute the Mean Random Consistency Index, $\operatorname{MRCI}(n)=\left(\bar{\lambda}_{\max }-n\right) /(n-1)$.

4) Compute the Consistency Ratio $\mathrm{CR}(n)=\mathrm{CI}(n)$ / $\operatorname{MRCI}(n)$ and evaluate the consistency, observing $\varepsilon=$ 0.052 for a $3 \times 3$ matrix; $\varepsilon=0.089$ for a $4 \times 4$ matrix; and $\varepsilon \simeq 0.1$ for a larger matrix.

- If $\operatorname{CR}(n)=0$ then $\mathbf{A}$ is consistent;

- If $\mathrm{CR}(n) / \mathrm{RI}(n) \leq \varepsilon$ then $\mathbf{A}$ is consistent;

- If $\mathrm{CR}(n) / \mathrm{RI}(n)>\varepsilon$ then $\mathbf{A}$ is not consistent. 


\section{F. Step 5}

Finally, the last step is to select the alternative that best satisfies the goal, and synthesize the priorities. This is done by multiplying vectors $\mathbf{w}^{\mathrm{C} k}$ and $\mathbf{w}^{\mathrm{A} k l}$ according

$$
\mathbf{w}^{\mathrm{D} k}=\left[w_{l}^{\mathrm{C} k} \cdot \mathbf{w}^{\mathrm{A} k l}\right]_{l} .
$$

The vectors $\mathbf{w}^{\mathrm{D} 1}, \mathbf{w}^{\mathrm{D} 2}$ and $\mathbf{w}^{\mathrm{D} 3}$ represent the ranking of the alternatives WLAN, UMTS and GPRS with regard to performance, cost and accessibility. Finally, the average ranking of these alternatives is computed as

$$
\mathbf{w}^{\mathrm{D}}=\frac{1}{3} \sum_{k=1}^{3} \mathbf{w}^{\mathrm{D} k} \text {. }
$$

The alternative with the largest value is then selected. This way of synthesizing the priorities is called the distributive mode [13].

\section{Metric Value Translations}

As pointed out before, the challenge of handling metric values (e.g originally from measurements) to a 9-point scale and subsequent determination of the value $a_{i j}$ arises.

Let our metric values be denoted as $\mathbb{Q}$ representing a set of numbers in decimal representation by computers. These numbers represent a physical metric value of some sort. The main goal is to translate the metric value into a scale suitable for a MCDM method.

Before the decision model is being used a path must be selected, for which there are four alternatives as shown in Fig. 3:

1) Path (a)-(f); the cognitive translation of a value which is directly fed into the AHP, and in this case a scale between 1 (equal importance) and 9 (extreme importance) is used. This step involves no mathematical tools;

2) Path (b)-(e); these metric values are translated with help from statistics before being fed into the AHP;

3) Path (b)-(d)-(f), going through statistics and a subsequent translation before entering the AHP. Such a translation can be a simple threshold-based one (cf. Subsection V.A) or based on FST (cf. Subsection V.B). In the latter case, the results are called "fuzzy quantities";

4) Path (c); the pure user perception and rating is fed into the AHP. This step involves no mathematical tools.

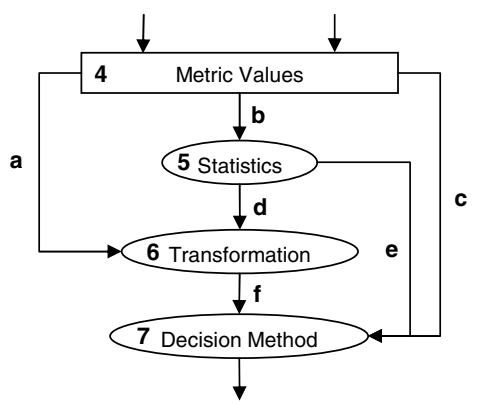

Fig. 3. Translations paths

\section{A. Example of a Simple Translation}

Each measured value is checked toward a set of thresholds. Tab. I shows a simple pseudo-code example in which the value $v$ is chosen based on the interval in which the number of packet losses per observation interval $\ell$ is found.

TABLE I

\begin{tabular}{|c|c|}
\hline 1 & if (networkType $==$ Network.WLAN) \\
\hline 3 & $\begin{array}{l}\text { if (packet_losses > 1) } \\
v=3 ;\end{array}$ \\
\hline 5 & $\begin{array}{l}\text { else if (packet_losses > 3) } \\
\quad v=5\end{array}$ \\
\hline 7 & $\begin{array}{l}\text { else if (packet_losses > 7) } \\
v=7 ;\end{array}$ \\
\hline 9 & $\begin{array}{l}\text { else } \\
v=1 ;\}\end{array}$ \\
\hline
\end{tabular}

METRIC VALUES FROM PACKET LOSSES

\section{B. Example of a Fuzzy Translation}

Let us again focus on $\ell$ as introduced in the last subsection. The particular coordinates for the translation based on the membership functions shown in Fig. 4 are given in Equation (5). Now, our metric values can be translated with the help of an irregular polygon into a 9-point scale to be used in the AHP decision tool.
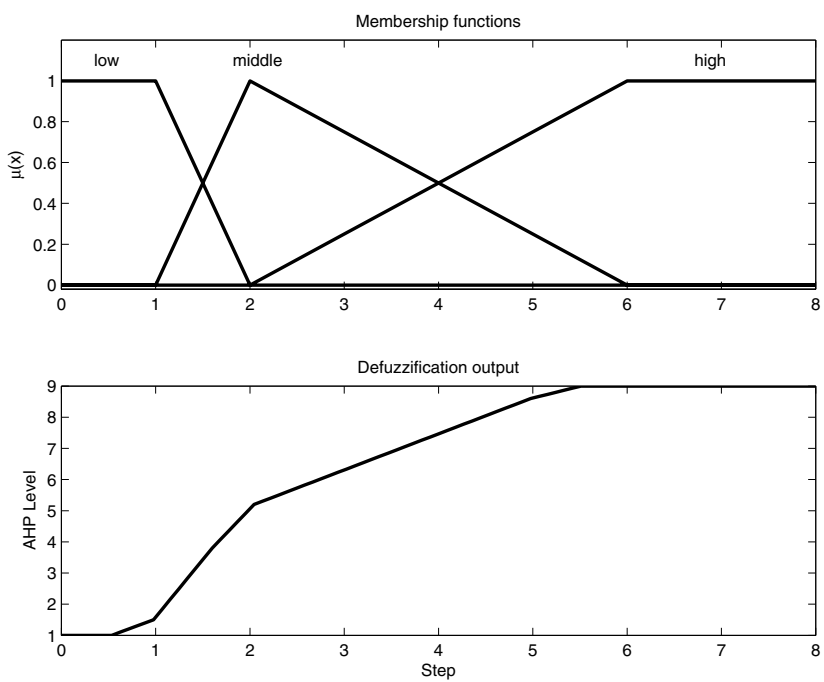

Fig. 4. Fuzzy Sets translations of packet losses

$$
v= \begin{cases}1.0 & \text { for } \ell<0.6 \\ \frac{1.5-1.0}{1.1-0.6} \cdot(\ell-0.6)+1.0 & \text { for } \quad 0.6 \leq \ell<1.1 \\ \frac{3.8-1.5}{1.8-1.1} \cdot(\ell-1.1)+1.5 & \text { for } 1.1 \leq \ell<1.8 \\ \frac{5.2-3.8}{2.3-1.8} \cdot(\ell-1.8)+3.8 & \text { for } \quad 1.8 \leq \ell<2.3 \\ \frac{8.6-5.2}{5.6-2.3} \cdot(\ell-2.3)+5.2 & \text { for } \quad 2.3 \leq \ell<5.6 \\ \frac{9.0-8.6}{6.2-5.6} \cdot(\ell-5.6)+8.6 & \text { for } \quad 5.6 \leq \ell<6.2 \\ 9.0 & \text { for } \quad 6.2 \leq \ell\end{cases}
$$




\section{Comparison}

We now focus at the processing of data coming from the above-described translation step, cf. link (f) in Fig. 3. The elements of an attribute matrix, as shown in Tab. II, $\mathbf{A}^{\mathrm{A} k l}$ are calculated as described in Tab. III based on the following comparisons: (1) WLAN-UMTS $\left(a_{12}^{\mathrm{A} k l}\right)$ and UMTS-WLAN $\left(a_{21}^{\mathrm{A} k l}\right)$; (2) WLAN-GPRS $\left(a_{13}^{\mathrm{A} k l}\right)$ and GPRS-WLAN $\left(a_{31}^{\mathrm{A} k l}\right)$; (3) UMTS-GPRS $\left(a_{23}^{\mathrm{A} k l}\right)$ and GPRS-UMTS $\left(a_{32}^{\mathrm{A} k l}\right)$. In the remainder of this subsection, the indexes ${ }^{\mathrm{A} k l}$ will be omitted.

TABLE II

A DECISION MATRIX WITH WLAN, UMTS AND GPRS

\begin{tabular}{l|c|c|c|} 
& WLAN & UMTS & GPRS \\
\hline WLAN & 1 & $a_{12}$ & $a_{13}$ \\
\hline UMTS & $a_{21}$ & 1 & $a_{23}$ \\
\hline GPRS & $a_{31}$ & $a_{32}$ & 1 \\
\hline
\end{tabular}

TABLE III

COMPARISON OF TRANSLATED VALUES.

\begin{tabular}{|c|c|}
\hline 1 & $\begin{array}{l}a=v_{1}-v_{2} ; / / \text { WLAN - UMTS } \\
\text { if }(a>=0)\end{array}$ \\
\hline 3 & $\begin{array}{l}a_{12}=a+1 \\
\text { else }\end{array}$ \\
\hline 5 & $\begin{array}{l}a_{12}=1 /(\operatorname{ABS}(a)+1) ; \\
a_{21}=1 / a_{12} ;\end{array}$ \\
\hline 9 & $\begin{array}{l}b=v_{1}-v_{3} ; / / \text { WLAN - GPRS } \\
\text { if }(b>=0)\end{array}$ \\
\hline 11 & $\begin{array}{l}a_{13}=b+1 \\
\text { else }\end{array}$ \\
\hline 13 & $\begin{array}{l}a_{13}=1 /(\operatorname{ABS}(b)+1) ; \\
a_{31}=1 / a_{13} ;\end{array}$ \\
\hline 15 & $\begin{array}{l}c=v_{2}-v_{3} ; / / \text { UMTS - GPRS } \\
\text { if }(c>=0)\end{array}$ \\
\hline 17 & $\begin{array}{l}a_{23}=c+1 \\
\text { else }\end{array}$ \\
\hline 19 & $\begin{aligned} a_{23} & =1 /(\operatorname{ABS}(c)+1) ; \\
a_{32} & =1 / a_{23} ;\end{aligned}$ \\
\hline
\end{tabular}

The comparison between WLAN and UMTS is represented by the local variable $a$, see Tab. III line 1. If the value is positive, the global variable $a_{12}$ is set to the calculated value as in line 3 . If the value is negative the global variable $a_{12}$ is set to the calculated value as in line 5. The opposite value is translated and saved as shown in line 6. These values are later used in the AHP. This is also done for the combinations WLAN-GPRS and UMTS-GPRS.

\section{CASE STUdY}

We consider a case study addressing two types of GS, a streaming service and a messaging service. Tab. IV shows the performance criteria settings in the matrix $\mathbf{A}^{\mathrm{C} 1}$. As a streaming service needs to behave well while in operation, the importance of the dynamic criteria (throughput, losses, RTT) is in general higher than the importance of the static ones, which is reflected in values $a_{i j}^{\mathrm{C} 1}<1$. Measured throughput and loss statistics are of similar importance $\left(a_{i 4}^{\mathrm{C} 1} \equiv a_{i 5}^{\mathrm{C} 1}\right.$ for $\left.i \leq 3\right)$ and much more relevant than the RTT $\left(a_{46}^{\mathrm{C} 1}=a_{56}^{\mathrm{C} 1}=5\right)$. Regarding cost (matrix $\mathbf{A}^{\mathrm{C} 2}$ ), a volume-based fee is much more critical than a time-based one $\left(a_{12}^{\mathrm{C} 2}=9\right)$. The relevance of the security overhead is considered to lie in-between that of the other two parameters $\left(a_{13}^{\mathrm{C} 2}=1 / 5, a_{23}^{\mathrm{C} 2}=5\right)$. Finally, the interference is considered as important as the coverage area (matrix $\mathbf{A}^{\mathrm{C} 3}$ with $a_{12}^{\mathrm{C} 3}=1$ ).

TABLE IV

COMPARISON OF PERFORMANCE CRITERIA FOR STREAMING SERVICE

\begin{tabular}{l|cccccc} 
& ID & LC & DL & Throughput & Losses & RTT \\
\hline ID & 1 & $1 / 5$ & $1 / 5$ & $1 / 7$ & $1 / 7$ & $1 / 3$ \\
LC & 5 & 1 & 1 & $1 / 5$ & $1 / 5$ & 1 \\
DL & 5 & 1 & 1 & $1 / 5$ & $1 / 5$ & 1 \\
Throughput & 7 & 5 & 5 & 1 & 1 & 5 \\
Losses & 7 & 5 & 5 & 1 & 1 & 5 \\
RTT & 3 & 1 & 1 & $1 / 5$ & $1 / 5$ & 1 \\
\multicolumn{4}{l}{$\lambda_{\max }=6.236, \mathrm{CI}=0.047, \mathrm{CR}=0.038$}
\end{tabular}

For a messaging service, things look different, cf. Tab. V. Here, the initial delay is of high to utmost importance $\left(a_{1 j}^{\mathrm{C1}}>\right.$ 1) due to the short-lived nature of the message delivery. The latter also makes a-priori measured static parameters much more relevant than dynamic parameters. For the same reason, capacity and throughput values are less important than losses and RTTs. Regarding cost, the time-based aspect is quite uncritical $\left(a_{12}^{\mathrm{C} 2}=9, a_{23}^{\mathrm{C} 2}=1 / 9\right)$, while the security overhead can be of particular importance $\left(a_{13}^{\mathrm{C} 2}=1 / 3\right)$. Again, interference is considered as important as the coverage area $\left(a_{12}^{\mathrm{C} 3}=1\right)$.

TABLE V

COMPARISON OF PERFORMANCE CRITERIA FOR MESSAGING SERVICE

\begin{tabular}{l|cccccc} 
& ID & LC & DL & Throughput & Losses & RTT \\
\hline ID & 1 & 7 & 3 & 7 & 5 & 3 \\
LC & $1 / 7$ & 1 & $1 / 5$ & 1 & $1 / 5$ & $1 / 7$ \\
DL & $1 / 3$ & 5 & 1 & 3 & 1 & $1 / 3$ \\
Throughput & $1 / 7$ & 1 & $1 / 3$ & 1 & $1 / 5$ & $1 / 5$ \\
Losses & $1 / 5$ & 5 & 1 & 5 & 1 & 1 \\
RTT & $1 / 3$ & 7 & 3 & 5 & 1 & 1 \\
\hline$\lambda_{\max }=6.293, \mathrm{CI}=0.059, \mathrm{CR}=0.047$ \\
\hline
\end{tabular}

The consistency ratios for both matrices shown in Tab. IV and $\mathrm{V}$ lie well below the critical upper limit of $10 \%$.

\section{A. Basic Scenario}

We now look at a scenario in which a streaming service has the possibility to use either WLAN, UMTS and GPRS. From the results in [11], we deduce the following static performancerelated matrices $\mathbf{A}^{\mathrm{A} k l}$ :

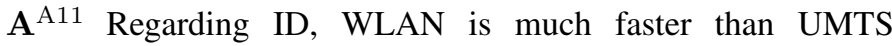
$\left(a_{12}^{\mathrm{A} 11}=7\right)$ and notably faster than GPRS $\left(a_{13}^{\mathrm{A} 11}=3\right)$. UMTS can be much slower than GPRS $\left(a_{23}^{\mathrm{A} 11}=1 / 5\right)$.

$A^{A 12}$ Regarding LC, WLAN offers much more bandwidth than UMTS $\left(a_{12}^{\mathrm{A} 12}=5\right)$, which in turn offers much more bandwidth than GPRS $\left(a_{23}^{\mathrm{A} 12}=5, a_{13}^{\mathrm{A} 12}=9\right)$. 
$\mathbf{A}^{\mathrm{A} 13}$ Regarding DL, WLAN is considered better than UMTS $\left(a_{12}^{\mathrm{A} 13}=3\right)$, and both outperform GPRS $\left(a_{13}^{\mathrm{A} 13}=\right.$ $\left.5, a_{23}^{\mathrm{A} 13}=3\right)$.

In the basic scenario, we assume that the monitoring does not display any performance problems. This means:

$\mathbf{A}^{\mathrm{A} 14}$ No particularities regarding throughput, i.e. all attributes are considered equal $\left(a_{12}^{\mathrm{A} 14}=1, a_{13}^{\mathrm{A} 14}=1\right.$ and $a_{23}^{\mathrm{A} 14}=$ $1)$.

$\mathbf{A}^{\mathrm{A} 15}$ The same holds for losses $\left(a_{12}^{\mathrm{A} 15}=1, a_{13}^{\mathrm{A} 15}=1\right.$ and $\left.a_{23}^{\mathrm{A} 15}=1\right)$.

A $^{\text {A16 }}$ RTT differ by nature; WLAN displays shorter RTT values as UMTS $\left(a_{12}^{\mathrm{A} 16}=5\right)$, which in turn outperforms GPRS $\left(a_{13}^{\mathrm{A} 16}=9, a_{23}^{\mathrm{A} 16}=5\right)$.

Looking at cost, we can state the following:

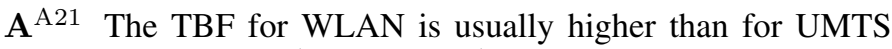
and GPRS $\left(a_{12}^{\mathrm{A} 21}=1 / 3, a_{13}^{\mathrm{A} 21}=1 / 3\right)$, which in turn use to be equally expensive $\left(a_{23}^{\mathrm{A} 21}=1\right)$.

A $^{\text {A22 }}$ Modern subscriptions imply flat rates, which is reflected in equal attributes $\left(a_{12}^{\mathrm{A} 22}=1, a_{13}^{\mathrm{A} 22}=1\right.$ and $\left.a_{23}^{\mathrm{A} 22}=1\right)$.

A $^{\text {A23 }}$ Overhead introduced by security has a lower impact for WLAN than for UMTS $\left(a_{12}^{\mathrm{A} 23}=3\right)$ and especially for GPRS $\left(a_{13}^{\mathrm{A} 23}=5, a_{23}^{\mathrm{A} 23}=3\right)$.

Regarding accessibility, we assume:

A $^{\text {A31 }}$ There is no coverage problem, which means that all attributes are considered equal $\left(a_{12}^{\mathrm{A} 31}=1, a_{13}^{\mathrm{A} 31}=1\right.$ and $\left.a_{23}^{\mathrm{A} 31}=1\right)$.

$\mathbf{A}^{\mathrm{A} 32}$ There is neither any problem with interference $\left(a_{12}^{\mathrm{A} 32}=\right.$ $1, a_{13}^{\mathrm{A} 32}=1$ and $a_{23}^{\mathrm{A} 32}=1$ ).

Tab. VI shows the vectors $\mathbf{w}^{\mathrm{D} 1}, \mathbf{w}^{\mathrm{D} 2}$ and $\mathbf{w}^{\mathrm{D} 3}$ as well as the average rating $\mathrm{w}^{\mathrm{D}}$ according to Eqn. (4). Performance shows the highest value for WLAN (0.438) as compared to UMTS and GPRS due to the outstanding static performance and the low RTT. Costs show the highest value for UMTS (0.349) compared to GPRS and WLAN due to the compromise between TBF and the limited impact of the overhead for security. Accessibility shows the same value (0.333) due to the same conditions for all technologies. Considering the average weights, the priority of choosing networks is (1) WLAN, (2) UMTS and (3) GPRS.

TABLE VI

RANKING For Streaming SERVICE IN BASIC Scenario

\begin{tabular}{l|ccc} 
& WLAN & UMTS & GPRS \\
\hline Performance & 0.438 & 0.298 & 0.264 \\
Cost & 0.311 & 0.349 & 0.340 \\
Accessibility & 0.333 & 0.333 & 0.333 \\
\hline Preference Ratio & 0.361 & 0.327 & 0.312
\end{tabular}

Let us now turn to the messaging service. As messages are short, the difference in link capacities is assumed to be negligible. This implies $a_{12}^{\mathrm{A} 12}=1, a_{13}^{\mathrm{A} 12}=1$ and $a_{23}^{\mathrm{A} 12}=1$. The remaining matrices $\mathbf{A}^{\mathrm{A} k l}$ are the same as for the streaming service.

Tab. VII shows the outcomes. Again, WLAN is considered outstanding regarding performance $(0.587)$, and UMTS wins the cost battle $(0.363)$. The average rating prioritizes WLAN before UMTS and GPRS. The latter are ranked quite similarly. The cost advantage for UMTS (smaller impact of the security overhead) is eaten up by worse performance mainly due to long IDs.

TABLE VII

RANKING For MESSAGING SERVICE IN BASIC SCENARIO

\begin{tabular}{l|ccc} 
& WLAN & UMTS & GPRS \\
\hline Performance & 0.587 & 0.189 & 0.224 \\
Cost & 0.320 & 0.363 & 0.317 \\
Accessibility & 0.333 & 0.333 & 0.333 \\
\hline Preference Ratio & 0.413 & 0.295 & 0.291
\end{tabular}

\section{B. Malfunctioning WLAN}

Let us now assume that our WLAN access experiences serious performance and accessibility problems due to heavy usage and interference in the $2.4 \mathrm{GHz}$ band. This is reflected in the following settings:

$\mathbf{A}^{\mathrm{A} 14}$ Very bad WLAN throughput $\left(a_{12}^{\mathrm{A} 14}=1 / 7, a_{13}^{\mathrm{A} 14}=1 / 3\right.$ and $\left.a_{23}^{\mathrm{A} 14}=1\right)$.

$\mathbf{A}^{\mathrm{A} 15}$ Many losses on the WLAN, which implies $a_{12}^{\mathrm{A} 15}=1 / 9$, $a_{13}^{\mathrm{A} 15}=1 / 9$ and $a_{23}^{\mathrm{A} 15}=1$.

$A^{\text {A16 }}$ RTT via WLAN in the order of that on UMTS: $a_{12}^{\mathrm{A} 16}=$ $1, a_{13}^{\mathrm{A} 16}=1 / 5, a_{23}^{\mathrm{A} 16}=5$.

$\mathbf{A}^{\mathrm{A} 31}$ Problems with WLAN coverage: $a_{12}^{\mathrm{A} 31}=1 / 5, a_{13}^{\mathrm{A} 31}=$ $1 / 5, a_{23}^{\mathrm{A} 31}=1$.

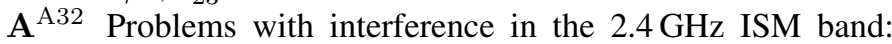
$a_{12}^{\mathrm{A} 32}=1 / 5, a_{13}^{\mathrm{A} 32}=1 / 5, a_{23}^{\mathrm{A} 32}=1$.

From Tab. VIII, we observe that WLAN dropped seriously in performance and that UMTS became best in all aspects for the streaming service. For the messaging service, see Tab. IX, UMTS and GPRS are almost equal, which again can be related to the better performance of GPRS regarding ID.

TABLE VIII

Ranking For Streaming Service, Malfunctioning WLAN

\begin{tabular}{l|ccc} 
& WLAN & UMTS & GPRS \\
\hline Performance & 0.216 & 0.430 & 0.354 \\
Cost & 0.319 & 0.347 & 0.334 \\
Accessibility & 0.091 & 0.455 & 0.455 \\
\hline Preference Ratio & 0.209 & 0.411 & 0.381
\end{tabular}

TABLE IX

RANKIng For Messaging Service, MALFunCtioning WLAN

\begin{tabular}{l|ccc} 
& WLAN & UMTS & GPRS \\
\hline Performance & 0.428 & 0.269 & 0.303 \\
Cost & 0.320 & 0.363 & 0.317 \\
Accessibility & 0.091 & 0.455 & 0.455 \\
\hline Preference Ratio & 0.280 & 0.362 & 0.358
\end{tabular}




\section{CONCLUSIONS}

In this paper, we have discussed and demonstrated the use of Multi-Criteria Decision Making (MCDM) based on the Analytic Hierarchy Process (AHP) for enabling a user being Always Best Connected (ABC) with regards to a set of criteria reflecting the needs of a Generic Service (GS). MCDM and AHP are typically used for management and business decisions in which the human aspect is involved to determine the importance between different criteria and alternatives. However, we have shown that the decision method is useful even for a technical application, namely to choose the appropriate connection between three alternatives WLAN, UMTS and GPRS in the context of seamless communications. We have seen that different criteria for different GS and conditions can make a difference regarding the decision based on performance, cost and accessibility ratings. As the calculations are neither numerous nor complicated, a use for real time link selection is possible. The proposed hierarchy can easily be adapted to own needs and preferences. It can also be expanded by additional criteria and attributes.

Future work includes refinements of the model such as introducing another decision level, yielding additional weights between performance, cost and accessibility. The model will also be connected to operational end-to-end monitoring and tested in real seamless communication scenarios.

\section{ACKNOWLEDGMENT}

The authors would like to thank the Swedish Agency for Innovation Systems VINNOVA for funding the project PIITSA = Personal Information for Intelligent Transport Systems through Seamless communications and Autonomous decisions (project number 2003-02873), which provided the context of this work.

\section{REFERENCES}

[1] M. Fiedler, S. Chevul, L. Isaksson, P. Lindberg, and J. Karlsson. Generic Communication Requirements of ITS-Related Mobile Services as Basis for Seamless Communications. In Proceedings of the First EuroNGI Conference on Traffic Engineering (NGI 2005), Rome, Italy, April 2005.

[2] L. Isaksson, M. Fiedler, and E. Rakus-Andersson. A Fuzzy Set Theory Based Method to Discover Transmissions in Wireless Personal Area Networks. In Proceedings of ICWMC'06, Rumänien, Bukarest, July 2006. ISBN 0-7695-2629-2.

[3] E. Gustafsson and A. Jonsson. Always Best Connected. IEEE Wireless Communications, 10(1):49-55, February 2003.

[4] B.S. Bakshi, P. Krishna, D.K. Pradhan, and N.H. Vaidya. Providing seamless communication in mobile wireless networks. In Proceedings 21st IEEE Conference on Local Computer Networks, pages 535-543, 13-16 Oct. 1996.

[5] J. Indulska and S. Balasubramaniam. Context-aware vertical handovers between WLAN and 3G networks. In IEEE 59th Vehicular Technology Conference, volume 5, pages 3019-3023, 17-19 May 2004.

[6] J.W. Floroiu, R. Ruppelt, D. Sisalem, and J. Voglimacci. Seamless handover in terrestrial radio access networks: a case study. IEEE Communications Magazine, 41(11):110-116, Nov. 2003.

[7] C. Åhlund, R. Brännström, and A. Zaslavsky. Traffic load Metrics for Multihomed Mobile IP and Gloabal Connectivity. In Telecommunication Systems, Springer Netherlands, 7 October, 2006.

[8] S. Chevul, L. Isaksson, M. Fiedler, P. Lindberg, and Roland Waltersson. Network Selection Box: An Implementation of Seamless Communication. In Proceedings of Third EuroNGI Workshop on Wireless and Mobility, Sitges, Spain, 7-9 June, 2005.
[9] J. Hou and D.C. O'Brien. Vertical handover-decision-making algorithm using fuzzy logic for the integrated Radio-and-OW system. In IEEE Transactions on Wireless Communications, volume 5, pages 176-185, Jan. 2006.

[10] S. Qingyang and A. Jamalipour. Network selection in an integrated wireless LAN and UMTS environment using mathematical modeling and computing techniques. IEEE Wireless Communications, 12(3):4248, June 2005.

[11] M. Fiedler, L. Isaksson, S. Chevul, J. Karlsson, and P. Lindberg. Measurements and Analysis of Application-Perceived Throughput via Mobile Links. In In Proceedings of the 2005 3ed Performance Modeling and Evaluation of Heterogeneous Networks (HET-NETs), page T06. Ilkley, West Yorkshire, U.K., 2005.

[12] T. Sutinen and T. Ojala. Case Study in Assessing Subjective QoS of a Mobile Multimedia Web Service in a Real Multi-Access Network. In Thirteenth International Workshop on Quality of Service. Passau, Germany, 2005.

[13] T.L. Saaty. The Analytic Hierarchy Process. McGraw-Hill, New York, 1980. ISBN 0-07-054371-2.

[14] D.R. Anderson, D.J. Sweeney, and T.A. Williams. Quantitative Methods for Business. Thompson/South-Western, Mason, OH, 9. edition, 2004.

[15] T.L. Saaty. How to make a decision: The Analytic Hierarchy Process. European Journal of Operational Research, 48(1):9-26, 1990.

[16] T.L. Saaty. Homogeneity and clustering in AHP ensures the validity of the scale. European Journal of Operational Research, 72:598-601, 1994.

[17] T.L. Saaty and L.G. Vargas. The Logic of Priorities. Kluwer Nijhoff Publishing, Boston, 1982. ISBN 0-89838-078-2.

[18] T.L. Saaty and M.S. Ozdemir. Negative Priorities in the Analytic Hierarchy process. Mathematical and Computer Modelling, 37:10631075, 2003

[19] T.L. Saaty and L.G. Vargas. Models, Methods, Concepts and Applications of the Analytic Hierarchy Process. Kluwer Academic Publishing, Dordrecht the Netherlands, 2001.

[20] M. Svahnberg, C. Wohlin, L. Lundberg, and M. Mattsson. A method for understanding quality attributes in software architecture structures. In SEKE '02: Proceedings of the 14th international conference on Software engineering and knowledge engineering, pages 819-826, New York, NY, USA, 2002. ACM Press.

[21] J. Karlsson and K. Ryan. A Cost-Value Approach for Prioritizing Requirements. IEEE Software, 14(5):67-74, Sep.-Oct. 1997.

[22] J. Karlsson, C. Wohlin, and B. Regnell. An evaluation of methods for prioritizing software requirements. Journal of Information and Software Technology, 39(14-15):939-947, 1998.

[23] M. Svahnberg. An industrial study on building consensus around software architectures and quality attributes. Information \& Software Technology, 46(12):805-818, 2004.

[24] InfoHarvest, Criterium DecisionPlus. http://www.infoharvest.com (Checked 2006-12-21).

[25] Arlingon Software Corporation, ERGO. http://www.arlingsoft.com (Checked 2006-12-21).

[26] Helsinki University of Technology, HIPRE+. http://www.sal.hut.fi/Downloadables/hipre3.html (Checked 2006-12-21).

[27] Krysalis Ltd., OnBalance. http://www.krysalis.co.uk (Checked 2006-12-21).

[28] Catalyze Ltd., Hiview. http://www.catalyze.co.uk (Checked 2006-12-21).

[29] T.L. Saaty and M.S. Ozdemir. Why the magic number seven plus or minus two. Mathematical and Computer Modeling, 38:233-244, 2003.

[30] G.A. Miller. The magical number seven, plus or minus two: Some limits on our capacity for processing information. Psychological Review, 63(2):81-97, 1956.

[31] R.T. Kellogg. Cognitive Psychology. SAGA Publications Ltd, London, 1997. ISBN 0-7619-5695-6. 